\title{
ASSAf consensus study on the ethical, legal and social implications of genetics and genomics in South Africa
}

AUTHORS:

Michael S. Pepper ${ }^{1,2}$

Collet Dandara ${ }^{3}$

Jantina de Vries ${ }^{4}$

Amaboo Dhai ${ }^{5}$

Melodie Labuschaigne ${ }^{6}$

Freddy Mnyongani ${ }^{7}$

Keymanthri Moodley ${ }^{8}$

Antonel Olckers ${ }^{9}$

Anne Pope ${ }^{10}$

Raj Ramesar ${ }^{11}$

Michele Ramsay ${ }^{12}$

Himla Soodyall13,14

Wayne Towers ${ }^{15}$

\section{AFFILIATIONS:}

1Institute for Cellular and

Molecular Medicine, Department of Immunology, Faculty of Health Sciences, University of Pretoria,

Pretoria, South Africa

${ }^{2}$ South African Medical Research Council Extramural Unit for Stem Cell Research and Therapy, University of Pretoria, Pretoria, South Africa

${ }^{3}$ Division of Human Genetics, Department of Pathology and Institute of Infectious Disease and Molecular Medicine, Faculty of Health Sciences, University of Cape Town, Cape Town, South Africa

${ }^{4}$ Department of Medicine, University of Cape Town, Cape Town, South Africa

${ }^{5}$ Steve Biko Centre for Bioethics, School of Clinical Medicine, Faculty of Health Sciences, University of the Witwatersrand, Johannesburg, South Africa

${ }^{6}$ Department of Jurisprudence, School of Law, University of South Africa, Pretoria, South Africa

${ }^{7}$ School of Law, University of KwaZulu-Natal, Durban, South Africa

${ }^{8}$ Centre for Medical Ethics and Law, Department of Medicine, Faculty of Health Sciences, Stellenbosch University, Stellenbosch, South Africa ${ }^{9}$ DNAbiotec (Pty) Ltd, Pretoria, South Africa

${ }^{10}$ Emeritus Associate Professor, Department of Private Law, Faculty of Law, University of Cape Town, Cape Town,
South Africa is home to one of the most genetically diverse populations in the world, which, combined with its high disease burden and high-quality infrastructure, makes our country a prime location for conducting genetics and genomics research. South African genomes are therefore highly sought after by the global research community. Increasingly, a range of technological advances, including the possibility of 'reading' whole genomes or exomes through next-generation sequencing, allows access to detailed molecular information from which information about health and disease can be inferred. This reading may also occur when information is collected for different purposes, which raises questions about the ethics of inferring information about health and disease in these situations. Against this backdrop of technological and ethical complexity, there is an urgent need to understand and protect the interests of patients and individuals who participate in research in the fields of genetics and genomics.

The publication of the sequence of the human genome 15 years ago heralded humankind's entry into a new era in which large volumes of data would be generated with the potential to drive major improvements in human health. A massive data management sector has evolved in parallel and includes data acquisition, storage, analysis, interpretation and access. This evolution of data has been accompanied by growth of commercial companies that utilise this information through the generation of products and services. Consequently, genetics and genomics present both opportunities and challenges in the South African context. Because of South Africa's history, during which segregation and oppression ruled - the effects of which are still experienced today - research as well as clinical and forensic practice in genetics and genomics raise many difficult issues that must be dealt with sensitively and constructively. Moreover, targeted policy, legislation, regulations or guidelines are lacking in these fields. For these reasons, in 2016, the Academy of Science of South Africa (ASSAf) undertook to conduct a consensus study on the ethical, legal and social implications (ELSI) of genetics and genomics work, as they relate to research, health service provision and forensic applications (medical and legal) in South Africa. The purpose of this study was to provide a well-researched document based on a combination of international best practices adapted to local conditions and deliberations by the panel, that will assist the national Departments of Health and Science and Technology to draft legislation, regulations and guidelines on matters pertaining to human genetics and the human genome. The study has been completed and a synopsis of some of the key issues and recommendations flowing from the consensus study is provided here.

The full report is divided into three thematic areas significant for the ELSI of genetics and genomics work: (1) building relationships, (2) respect for persons and (3) good stewardship. Following analysis of each theme, pragmatic, ethically and legally sound, culturally appropriate, feasible, enforceable and sustainable recommendations are proposed to optimise use of resources in the country. All recommendations are intended to underpin dialogue and discussions that must lead to new policy, legislation, regulations and guidelines.

Given that genetic and genomic information has implications for individuals, families and communities, the report looks at the ELSI of genetics and genomics in South Africa through the lens of the communitarian ubuntu philosophy, loosely translated as 'humanity', or more commonly 'I am because we are', which continues to drive a South African national consciousness in the process of democratic transformation. Ubuntu deepens respect for persons and pervades every sphere of South African life, including science. The degree to which ubuntu is practised consciously may differ in urban and rural communities. It may also differ amongst youth and older members of society. In this regard, it may be said that relative solidarity is an important component of ubuntu. That it is increasingly pervasive is illustrated by the observation that the South African judiciary has embraced ubuntu as an integral part of the constitutional values and principles, especially when interpreting the Bill of Rights. The panel therefore recommends that the ubuntu principle must be promoted in genetics and genomics research, healthcare delivery and forensics practice (Recommendation 5). Although the notions of personal autonomy and ubuntu may appear to be in tension, they should be seen to be complementary rather than mutually exclusive. Ubuntu does not negate individual choice or the exercise of autonomy; rather, individual choice is expected to take the community context into account. Genetics and genomics are good examples of why ubuntu should infuse our decision-making, because of the inherent 'domino-effect' of those choices on families and communities.

The section on 'Building Relationships' focuses on engagement between human genetics/genomics practitioners and the general public and communities, and recommends that close attention is given to stakeholder engagement (Recommendation 1) to promote understanding amongst all role players about their roles and responsibilities. Topics for engagement range from academic research projects, to genetic testing in the public and private sectors, and also include the relationships among the public, the criminal justice system and the forensic science sector of the country. Accountability and transparency are emphasised for all aspects (Recommendation 4). Further, the report highlights South African experiences with community engagement for genomics and the critical importance of education and translation of science into policy and practice (Recommendation 2). Regular evaluation of the effectiveness of stakeholder engagements is also recommended.

This section also emphasises the importance of ensuring that the public is well informed about participating in research projects, as well as their roles, rights and responsibilities when choosing to use direct-to-consumer genetic marketing and testing approaches (Recommendations 3 and 8 ). Regarding direct-to-consumer marketing, the advent of social media and the ease with which information is transmitted have facilitated access to large 
South Africa

${ }^{11} \mathrm{MRC} /$ UCT Research Unit for Genomic and Precision Medicine, Division of Human Genetics, Institute of Infectious Diseases and Molecular Medicine, University of Cape Town, Cape Town, South Africa ${ }^{12}$ Sydney Brenner Institute for Molecular Bioscience, Faculty of Health Sciences, University of the Witwatersrand, Johannesburg, South Africa

${ }^{13}$ Division of Human Genetics, School of Pathology, Faculty of Health Sciences, University of the Witwatersrand, Johannesburg, South Africa

${ }^{14}$ National Health Laboratory Service, Johannesburg, South Africa

${ }^{15}$ Africa Unit for Transdisciplinary Health Research (AUTHeR), North-West University, Potchefstroom, South Africa

\section{CORRESPONDENCE TO:} Michael Pepper

EMAIL:

michael.pepper@up.ac.za

\section{KEYWORDS:}

regulation; stewardship; ubuntu; health care; privacy

\section{HOW TO CITE:}

Pepper MS, Dandara C, De Vries J, Dhai A, Labuschaigne $\mathrm{M}$, Mnyongani $\mathrm{F}$, et al. ASSAf consensus study on the ethical, legal and social implications of genetics and genomics in South Africa. S Afr J Sci. 2018;114(11/12), Art. \#a0302, 3 pages. https://dx.doi. org/10.17159/sajs.2018/a0302

\section{PUBLISHED:}

27 November 2018 amounts of information on the possible consequences of genomic variants on health and disease for individuals and their families. This desire for information is driving an industry in which individuals can obtain very detailed information on their own genomes simply by providing a biological sample (e.g. sputum or a cheek swab) and mailing it to an entity that will do the testing and analysis. Although this practice promotes the exercise of autonomy to access meaningful personal information, the process could impact negatively on the person depending on (1) the quality of the test and (2) the means by which results and the implications of the findings are delivered, which should be through direct counselling. Negative effects create anxiety and confusion, as the recipient may not know how to respond to the information. Secondly, not all genetic/genomic variants have consequences for health and disease. To protect the public, the panel recommends that direct-to-consumer genetic marketing and testing must be regulated (Recommendation 3). The panel also recommends that the South African Health Products Regulatory Authority (SAHPRA) should include regulation of genetic tests under the Medical Devices Act (No. 14 of 2015) (Recommendation 13).

The topic 'Respect for Persons' is addressed in light of the Constitution, which recognises and protects fundamental individual rights as well as cultural and communal interests, including protecting the confidentiality of personal information and access to and control over such information. Legally and ethically, people are entitled to make informed choices about their health care and research participation. Especially relevant are privacy interests as genetic information could theoretically be a means to identify a person, even if data are de-identified. The report recommends that the existing legal and ethical framework relating to the protection of personal information, access to, control over and use of personal information and data be revised to provide for a well-aligned and integrated framework that balances a range of diverse interests. The Protection of Personal Information Act (No. 4 of 2013) (POPI) provides some guidance on how to manage disclosure and sharing of personal information. The panel recommends that 'Engagement with the Information Regulator, the Department of Justice and Constitutional Development, is important to discuss the development of regulations in the POPI Act (No. 4 of 2013) and how this will impact on genetics and genomics research' (Recommendation 7). Informed consent is required, as usual, for genetics and genomics work, whether for diagnostic, therapeutic or research purposes. The consensus report endorses the current Department of Health's Ethics in Health Research Guidelines (2015) which advocates for broad, tiered or specific consent, according to the study protocol and the choice of the participant or sample provider. The panel recognises, however, that there is lack of consensus regarding the impact of the POPI Act on broad consent and that the situation may change once the Act is fully operational and clarity is obtained from the Regulator. The report recommends that a national consent template for genetics and genomics would be useful so that the essential considerations are not omitted (Recommendation 6).

The third thematic area, 'Good Stewardship', emphasises the inherent characteristics of integrity, honesty, accountability and sharing that inform the notion of stewardship. Policies, legislation, regulations and guidelines must govern genetic and genomic testing; accreditation of laboratories; qualification and certification of staff; and mechanisms and circumstances for feedback of individual results, especially incidental findings. National frameworks for biobanks and access to samples and data are necessary to promote equitable and responsible sharing that will enhance knowledge generation and translational science while aligning with international policies and guidelines (Recommendation 10).

Matters pertaining to ownership/custodianship of tissue, DNA and other biological samples, particularly in the context of secondary use of data and samples, as well as benefit sharing, remain contentious and continue to stimulate debate. The panel suggests that these matters should be considered in particular by the South African Law Reform Commission and the National Intellectual Property Management Office. It is recommended that the country should debate, explore and adapt the recommended 'sociologically informed model' to identify principles of custodianship/ownership of samples and benefit sharing, because the topics have a cascade of implications: ethical values of equity and distributive justice; good governance principles of benefit sharing; and whether intellectual property can exist if genomic resources are to be regarded as a 'common good' (Recommendations 12 and 18).

Given the complexity and rapid evolution of the fields of genetics and genomics, the panel suggests that a 'South African Human Genetics Advisory Board should be established' (Recommendations 11 and 16). The recommendation is that 'The Board should have appropriate expertise to provide guidance to policymakers and regulatory structures'. Furthermore, 'The South African Human Genetics Advisory Board (SAHGB) should be adequately resourced and independent, with the aim of providing oversight in genetics and genomics at the national level and working in concert with ethics and legal regulatory structures'.

One of the most significant overarching recommendations concerns the need to develop capacity in human and material resources. This includes in particular, technical, scientific, computational, bioinformatics and statistical analysis, as well as financial, legal and ethical expertise. Training of genetics nurses, genetics counsellors, medical geneticists, medical scientists, bioinformaticists, biostatisticians and forensic scientists for the public and private sectors is critical to provide a service platform and sustainable genomics programmes to benefit the nation (Recommendation 14). The United Kingdom has committed to their programme of 'Genomics England' within which the concept of workforce training has been firmly embedded (https://www.genomicsengland.co.uk/aboutgecip/for-gecip-members/education-and-training/), and this could be a model for the development of genomics in South Africa. The panel recommends that effective measures be implemented to 'improve the public's knowledge and understanding of genetics, genomics and associated new technologies in a culturally sensitive and appropriate manner'; to raise awareness of predatory practices where and when they arise; and to educate the public. The Departments of Basic Education and Higher Education and Training should be encouraged to integrate education about new health-related technologies into primary, secondary and tertiary education curricula. Appropriate genetics and genomics training should be promoted for healthcare professionals, and a substantive investment should be 
made into 'training of genetic counsellors and clinical geneticists and other relevant professionals to increase the national capacity to deliver genetics and genomics services' (Recommendation 2). The panel also suggests that courses on forensic DNA testing should be incorporated into the curricula of law degrees.

A strong legal and ethical framework is required that includes: review and oversight roles for research ethics committees and data access committees; engagement with the Information Regulator and the Department of Justice; as well as clear expectations about avoiding harm or offence when reporting research findings (Recommendations 7 and 17). In order to ensure that professionals working in the fields of genetics and genomics adhere to the highest standards of practice, the panel highlights the need for responsibility and a code of conduct (Recommendation 9) regarding sustainable and careful use of genomic resources (reflected as both a value and a practice) by individuals, communities, organisations, companies and governmental institutions. This code of conduct could be established in consultation with professional councils like the Health Professionals Council of South Africa and the Nursing and Pharmacy Council as well as other appropriate professional boards/councils or regulatory bodies, and should be aligned with values and principles from international best practice. It is also necessary to ensure that sanctions for non-compliance with requirements exist and can be enforced. Consequently, authority to impose and enforce sanctions must be allocated appropriately, and the panel recommends that 'Sanctions for non-compliance with current and future legislation must be defined, be implementable and be effective' (Recommendation 19).

Finally, when accessing personal information (demographic, phenotypic and genotypic - which potentially could be used to identify a person), individuals must be protected to avoid social harm such as negative perceptions, stigmatisation and discrimination. In addition, participants must be given the option to gain access to this information, although which information should be made available and to which degree, as well as the way in which information is provided, remain controversial and continue to be debated. Likewise, ownership of data and samples remains a contested issue and researchers must be aware that they are custodians of data and samples, rather than legal owners thereof.

In summary, the consensus report emphasises the benefits to be derived from genetics and genomics work in research, clinical practice and forensic science, and the need for boundaries to be clearly defined and policies adhered to so that the benefits are shared by all while avoiding unnecessary harm. From a genetics and genomics perspective, material differences exist between individuals and between different groups of people. Tools are needed to utilise these differences to better manage prevention, diagnosis and treatment of disease. However, it is increasingly recognised that the analysis of findings for an individual affects not only the immediate biological family members directly (through transmission of heritable traits as genetic information is both personal and familial at the same time), but also indirectly the community to which an individual belongs. Sensitive findings may provoke emotional reactions; negative perceptions about findings can therefore affect large numbers of people, and this should be considered in the regulation of genetics and genomics for the country. Laws and regulations relating to genetics and genomics must be aligned and consistent, and where necessary drafted or updated from time to time to remain abreast of new developments in the field. Ultimately, the practice of genetics and genomics should serve the people of South Africa in the spirit of ubuntu.

\section{Acknowledgements}

This consensus study was undertaken under the auspices of the Academy of Science of South Africa, and the panel expresses heartfelt thanks to Prof. Roseanne Diab and Dr Khutso Phalane for all they have done to ensure the successful outcome of the project. We are also grateful to the Department of Science and Technology for the funding provided. 